International Journal of Physical Research, $8(1)(2019) 14-21$
International Journal of Physical Research
WPC
Website: www.sciencepubco.com/index.php/IJPR
Research paper

\title{
Exploration on traveling wave solutions to the 3rd-order klein-fock-gordon equation (KFGE) in mathematical physics
}

\author{
Nur Hasan Mahmud Shahen ${ }^{1}{ }^{*}$, Foyjonnesa ${ }^{1}$, Md. Habibul Bashar ${ }^{1}$ \\ ${ }^{1}$ European University of Bangladesh, Dhaka-1216, Bangladesh \\ *Corresponding author E-mail: nhmshahen_math@eub.edu.bd
}

\begin{abstract}
In this paper, the -expansion method has been applied to find the new exact traveling wave solutions of the nonlinear evaluation equations (NLEEs) by utilizing 3rd-order Klein-Gordon Equation (KFGE). With the collaboration of symbolic commercial software maple, the competence of this method for inventing these exact solutions has been more exhibited. As an upshot, some new exact solutions are obtained and signified by hyperbolic function solutions, different combinations of trigonometric function solutions, and exponential function solutions. Moreover, the -expansion method is a more efficient method for exploring essential nonlinear waves that enrich a variety of dynamic models that arises in nonlinear fields. All sketching is given out to show the properties of the innovative explicit analytic solutions. Our proposed method is directed, succinct, and reasonably good for the various nonlinear evaluation equations (NLEEs) related treatment and mathematical physics also.
\end{abstract}

Keywords: The Expansion Method; Nonlinear Evolution Equation; Exact Solution; 3rd-Order Klein-Gordon Equation; Mathematical Physics.

\section{Introduction}

Physical phenomena and processes that occurred in nature generally have tangled nonlinear features. Nonlinear problems (NLPs) are of interest to engineers, biologists, physicists, mathematicians, and many other scientists because most systems are inherently nonlinear in nature. From there nonlinear evaluation equations have been the topic of concern in different branches of nonlinear sectors such as physics, optical fibers, plasma physics, neural physics, solid-state physics, propagation of shallow water wave, mathematical fluid dynamics, electromagnetism, signal processing, chaos, viscoelasticity, heat flow and wave propagation phenomena, applied mathematics, protein chemistry, geochemistry, chemical kinematics, chemically reactive materials and meteorology, etc. That is why the exploration of traveling wave solutions is becoming a matter of concerning issue day by day.

Our proposed Klein-Fock-Gordon equation (KFGE) is an important class of NLEEs that arise in the theory of relativity, relativistic quantum mechanics and quantum field theory, which is also of great significance for the high energy particle physics and is applied to model various types of matter, including the spread of deviation in crystals and the properties of elementary particles. Sometimes it is defined as the equation of relativistic wave related to Schrodinger equation.

Herewith the Klein-Fock-Gordon equation (KFGE) [32] is defined as the form

$\frac{\partial^{2} u}{\partial t^{2}}+\alpha \frac{\partial^{2} u}{\partial x^{2}}+\beta u+\partial u^{m}=f(x, t)$

In this work, we mainly study a special case of nonlinear Klein-Fock-Gordon equation by using $\mathrm{m}=3$ in Eq. (1).This $\mathrm{m}^{\text {th }}$ Order KleinFock-Gordon equations Eq. (1) has an $\mathrm{m}^{\text {th }}$ order source term, a linear source term, and a $2^{\text {nd }}$ order derivative in the initial value variable and spatial variables. When $\mathrm{m}=2$ the equation is called the $2^{\text {nd }}$ order or quadratic Klein-Fock-Gordon equation and when $\mathrm{m}=3$ the equation is tern into the cubic or $3^{\text {rd }}$ order Klein-Fock-Gordon equation.

The main goal of this article is to apply the $\exp (-\varphi(\xi))$-expansion method to find the exact solutions of nonlinear $3^{\text {rd }}$ order Klein-FockGordon equation.

Nowadays, the exact traveling wave solution for nonlinear evaluation equations (NEEs) has been explored by many authors and they have been used many powerful methods. Many powerful method have been presented such as Hirota's bilinear transformation method [1], [2], The $\exp (-\varphi(\xi))$-expansion method[3-5], The exp-function expansion method [6], The Extend $\exp (-\varphi(\xi))$-expansion method [7], [8], The extended tanh-function method [9], [10], Lie symmetry method [11-14], The modified simple equation method [15], [16], The complex hyperbolic function method [17], The Bernoulli's Sub-ODE method [18], The extended sinh-cosh and sin-cos methods [19], The (G'/G)- 
expansion method [20-22], The enhance (G'/G)-expansion method [23], The Jacobi elliptic function method [24], Homogeneous balance method [25, 26], He's polynomial [27], Asymptotic methods and Nano mechanics [28], The extended multiple Riccati equations expansion method [29], The variational iteration method [30] and so on.

M.G. Hafez [31] explored the coupled Klein-Gordon-Zakharov equation using $\exp (-\varphi(\xi))$-expansion method but he didn't discuss the 3rd-Order or cubic Klein-Fock-Gordon equations. For this, we firmly intended our self to solve the 3rd-Order or cubic Klein-Fock-Gordon equations for the better solution of mathematical and physical treatment.

The rest of this article is prepared as follows: In section 2, the $\exp (-\varphi(\xi))$-expansion method has been discussed. In section 3, we applied this method to the proposed nonlinear evolution equations pointed out above. In section 4, we provide some graphical representations among the obtained solutions. Finally, conclusions are given in section 5.

\section{Formation of the $\exp (-\varphi(\xi))$-expansion method:}

In this section, we will describe $\exp (-\varphi(\xi))$-expansion method by term. Let us consider a nonlinear partial differential equation in the following form,

$\Re\left(U, U_{x x}, U_{x z}, U_{x x}, U_{x y}, U_{x t t}, \ldots \ldots\right)=0$.

Where $U=U(x, y, z, t)$ is an unknown function, $\Re$ is a polynomial of $U$ and its different type partial derivatives, in which the nonlinear terms and the highest order derivatives are involved.

Step-1. Now we consider a transformation variable to convert all independent variable into one variable, $\operatorname{such}$ as $U(x, t)=u(\xi)$,

$\xi=k x+l y+m z \pm V t$.

By implementing this variable Eq. (3) permits us reducing Eq. (2) in an ODE for $u(x, t)=u(\xi)$

$$
P\left(u, u^{\prime}, u^{\prime \prime}, \cdots \cdots \cdots\right)=0
$$

Step-2. Suppose that the solution of ODE Eq. (4) can be expressed by a polynomial in $\exp (-\varphi(\xi))$ as follows

$$
u=\sum_{i=0}^{m} a_{i} \exp (-\varphi(\xi))^{i}
$$

Where the derivative of $\varphi(\xi)$ satisfies the ODE in the following form

$$
\varphi^{\prime}(\xi)=\exp (-\varphi(\xi))+\mu \exp (\varphi(\xi))+\lambda .
$$

Then the solutions of ODE Eq. (7) are

Case I:

Hyperbolic function solution (when $\lambda^{2}-4 \mu>0, \mu \neq 0$ ):

$\varphi(\xi)=\ln \left(\frac{-\sqrt{\lambda^{2}-4 \mu} \tanh \left(\frac{\sqrt{\lambda^{2}-4 \mu}}{2}(\xi+C)\right)-\lambda}{2 \mu}\right)$.

And

$\varphi(\xi)=\ln \left(\frac{-\sqrt{\lambda^{2}-4 \mu} \operatorname{coth}\left(\frac{\sqrt{\lambda^{2}-4 \mu}}{2}(\xi+C)\right)-\lambda}{2 \mu}\right)$.

Case II:

Trigonometric function solution (when $\lambda^{2}-4 \mu<0, \mu \neq 0$ ):

$\varphi(\xi)=\ln \left(\frac{\sqrt{4 \mu-\lambda^{2}} \tan \left(\frac{\sqrt{4 \mu-\lambda^{2}}}{2}(\xi+C)\right)-\lambda}{2 \mu}\right)$. 
$\varphi(\xi)=\ln \left(\frac{\sqrt{4 \mu-\lambda^{2}} \cot \left(\frac{\sqrt{4 \mu-\lambda^{2}}}{2}(\xi+C)\right)-\lambda}{2 \mu}\right)$

Case III:

Exponential function solution (when $\lambda^{2}-4 \mu>0, \mu=0$ ):

$\varphi(\xi)=-\ln \left(\frac{\lambda}{\exp (\lambda(\xi+C))-1}\right)$

Case IV:

Rational function solution (when $\lambda^{2}-4 \mu=0, \mu \neq 0, \lambda \neq 0$ ):

$\varphi(\xi)=\ln \left(-\frac{2(\lambda(\xi+C)+2)}{\lambda^{2}(\xi+C)}\right)$

Case V:

Other solution (when $\lambda^{2}-4 \mu=0, \mu=\lambda=0$ ):

$\varphi(\xi)=\ln (\xi+C)$.

Where $a_{i}, V, \lambda ; i=0, \cdots \cdots, \mathrm{m}$ and $\mu$ are constants to be determined later. The positive integer $\mathrm{m}$ can be determined by considering the homogeneous balance between the highest order derivatives and nonlinear terms appearing in ODE (3).

Step-3. By substituting Eq. (5) into Eq. (4) and using the ODE (6), collecting all same order of $\exp (-\varphi(\xi))$ together, then we execute an polynomial form of $\exp (-\varphi(\xi))$. Equating each coefficients of this polynomial to zero, yields a set of algebraic system for $a_{i}, \cdots V, \lambda ; i=0, \cdots \cdots, \mathrm{m}$ and $\mu$.

Step-4. Assuming that the constants $a_{i}, \cdots V, \lambda ; i=0, \cdots \cdots, \mathrm{m}$ and $\mu$ can be obtained by solving the algebraic system, since the general solutions of the auxiliary ODE (6) have been well known for us, then substituting $a_{i}, \cdots V ; i=0, \cdots \cdots, \mathrm{m}$, and the general solutions of Eq.(5) into Eq.(6). Thus we attain exact and explicit traveling wave solutions of nonlinear partial differential equation (2).

\section{Application of the suggested method}

In this section we apply our proposed $\exp (-\varphi(\xi))$-expansion method to find the exact solution of cubic Klein-Fock-Gordon equation. Here for our convenient we consider $m=3$ to the $m^{\text {th }}$ order Klein-Fock-Gordon equation [31].

$u_{t t}+\alpha u_{x x}+\beta u+\delta u^{3}=f(x, t)$

where $\alpha, \beta, \delta$ are nonzero constants.

The travelling wave equation is of the form

$u=u(x, t), \quad \xi=m x-b t, \quad u=u(\xi), \quad u(x, t)=u(\xi)$

Using travelling wave equation Eq. (8) and integrating Eq. (7) with respect to $\xi$, we obtain the following ordinary differential equation.

$\left(b^{2}+\alpha m^{2}\right) u^{\prime \prime}+\beta u+\delta u^{3}=0$.

Now considering the homogeneous balance between the highest order derivative $u^{\prime \prime}$ and nonlinear term $u^{3}$, then we get $n=1$. Therefore, our suggested method allows us to use the auxiliary solution in the following form:

$$
u(\xi)=A_{0}+A_{1} e^{-\varphi(\xi)}
$$

Where $A_{0}$ and $A_{1}$ are arbitrary constant to be determined such that $A_{1} \neq 0$, while $\lambda, \mu$ are arbitrary constants.

Now differentiating Eq. (10) and using Eq. (6) we get

$u^{\prime \prime}(\xi)=-A_{1}(-(\exp (-\varphi(\xi))+\mu \exp (\varphi(\xi))+\lambda) \exp (-\varphi(\xi))+\mu(\exp (-\varphi(\xi))+\mu \exp (\varphi(\xi))+\lambda) \exp (\varphi(\xi))) \exp (-\varphi(\xi))$

$+A_{1}(\exp (-\varphi(\xi))+\mu \exp (\varphi(\xi))+\lambda)^{2} \exp (-\varphi(\xi))$

And also calculate $u^{3}(\xi)=A_{0}^{3}+\frac{3 A_{0}^{2} A_{1}}{\exp (\varphi(\xi))}+\frac{3 A_{0} A_{1}^{2}}{(\exp (\varphi(\xi)))^{2}}+\frac{A_{1}^{3}}{(\exp (\varphi(\xi)))^{3}}$. 
Now putting the value of $u, u^{\prime \prime}, u^{3}$ in Eq. (9) and coefficient of $e^{i \varphi(\xi)}, i=0, \pm 1, \pm 2, \cdots \cdots$, to zero, we get

$$
\begin{aligned}
& A_{1} \mu \lambda \alpha m^{2}+A_{1} \mu \lambda b^{2}+\delta A_{0}^{3}+\beta A_{0}=0 . \\
& \alpha \lambda^{2} m^{2} A_{1}+2 \alpha m^{2} \mu A_{1}+b^{2} \lambda^{2} A_{1}+2 b^{2} \mu A_{1}+3 \delta A_{0}^{2} A_{1}+\beta A_{1}=0 . \\
& 3 \alpha \lambda m^{2} A_{1}+3 b^{2} \lambda A_{1}+3 \delta A_{0} A_{1}^{2}=0 . \\
& 2 \alpha m^{2} A_{1}+\delta A_{1}^{3}+2 b^{2} A_{1}=0 .
\end{aligned}
$$

Solving the polynomial (11)-(14) by using maple we get the following sets:

Set-1:

$$
b= \pm \sqrt{\frac{\alpha m^{2} \lambda^{2}+4 \alpha m^{2} \mu-2 \beta}{\lambda^{2}-4 \mu}}, \quad m=m, A_{0}= \pm \frac{\beta \lambda}{\left(\lambda^{2}-4 \mu\right) \delta \sqrt{-\frac{\beta}{\delta \lambda^{2}-4 \delta \mu}}}, A_{1}= \pm 2 \sqrt{-\frac{\beta}{\delta \lambda^{2}-4 \delta \mu}}
$$

Set-2:

$$
b= \pm \sqrt{\frac{\alpha m^{2} \lambda^{2}+4 \alpha m^{2} \mu-2 \beta}{\lambda^{2}-4 \mu}}, \quad m=m, A_{0}=\frac{\beta \lambda}{\left(\lambda^{2}-4 \mu\right) \delta \sqrt{-\frac{\beta}{\delta \lambda^{2}-4 \delta \mu}}}, A_{1}=-2 \sqrt{-\frac{\beta}{\delta \lambda^{2}-4 \delta \mu}},
$$

Where $\mu$ and $\lambda$ are arbitrary constants.

Now substituting the values of $b, m, A_{0}, A_{1}$ into Eq. (10) we get

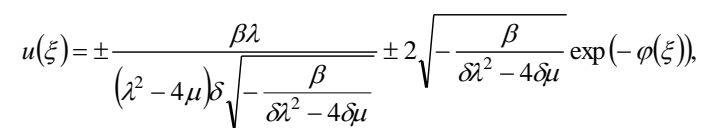

Where, $\xi=-\sqrt{\frac{\alpha m^{2} \lambda^{2}+4 \alpha m^{2} \mu-2 \beta}{\lambda^{2}-4 \mu}} t+m x$.

Case-I: (when $\lambda^{2}-4 \mu>0, \mu \neq 0$ ) we get following hyperbolic solution

Family-1

$$
\begin{aligned}
& u_{1,2}(\xi)= \pm \frac{\beta \lambda}{\left(\lambda^{2}-4 \mu\right) \delta \sqrt{-\frac{\beta}{\delta \lambda^{2}-4 \delta \mu}}}+\frac{2 \pm 2 \sqrt{-\frac{\beta}{\delta \lambda^{2}-4 \delta \mu}} \mu}{-\sqrt{\lambda^{2}-4 \mu} \tanh \left(\frac{1}{2} \sqrt{\left.\left(\lambda^{2}-4 \mu\right)(\xi+C)\right)-\lambda}\right.}, \\
& u_{3,4}(\xi)= \pm \frac{\beta \lambda}{\left(\lambda^{2}-4 \mu\right) \delta \sqrt{-\frac{\beta}{\delta \lambda^{2}-4 \delta \mu}}}+\frac{2 \pm 2 \sqrt{-\frac{\beta}{\delta \lambda^{2}-4 \delta \mu}} \mu}{\sqrt{-\lambda^{2}+4 \mu} \tan \left(\frac{1}{2} \sqrt{\left(-\lambda^{2}+4 \mu\right)}(\xi+C)\right)-\lambda},
\end{aligned}
$$

Where, $\xi=-\sqrt{-\frac{\alpha m^{2} \lambda^{2}-4 \alpha m^{2} \mu-2 \beta}{\lambda^{2}-4 \mu}} t+m x$, and $C$ is an arbitrary constant.

Case-II: (when $\lambda^{2}-4 \mu>0, \quad \mu \neq 0$ ) get following trigonometric solution Family-2

$$
\begin{aligned}
& u_{5,6}(\xi)= \pm \frac{\beta \lambda}{\left(\lambda^{2}-4 \mu\right) \delta \sqrt{-\frac{\beta}{\delta \lambda^{2}-4 \delta \mu}}}+\frac{2 \pm 2 \sqrt{-\frac{\beta}{\delta \lambda^{2}-4 \delta \mu}} \mu}{-\sqrt{\lambda^{2}-4 \mu} \operatorname{coth}\left(\frac{1}{2} \sqrt{\left(\lambda^{2}-4 \mu\right)}(\xi+C)\right)-\lambda}, \\
& u_{7,8}(\xi)= \pm \frac{\beta \lambda}{\left(\lambda^{2}-4 \mu\right) \delta \sqrt{-\frac{\beta}{\delta \lambda^{2}-4 \delta \mu}}}+\frac{2 \pm 2 \sqrt{-\frac{\beta}{\delta \lambda^{2}-4 \delta \mu}} \mu}{\sqrt{-\lambda^{2}+4 \mu} \cot \left(\frac{1}{2} \sqrt{\left.\left(-\lambda^{2}+4 \mu\right)(\xi+C)\right)-\lambda}\right.},
\end{aligned}
$$


Where, $\xi=-\sqrt{-\frac{\alpha m^{2} \lambda^{2}-4 \alpha m^{2} \mu-2 \beta}{\lambda^{2}-4 \mu}} t+m x$, and $C$ is an arbitrary constant.

Case-III: (when $\lambda^{2}-4 \mu>0, \quad \mu=0, \quad \lambda \neq 0$ ) we get following exponential solution

Family-3

$u_{9,10}(\xi)= \pm \frac{\beta}{\lambda \delta \sqrt{-\frac{\beta}{\delta \lambda^{2}}}}-\frac{1}{2} \frac{ \pm 2 \sqrt{-\frac{\beta}{\delta \lambda^{2}}} \lambda^{2}(\xi+C)}{\lambda(\xi+C)+2}$,

Where, $\xi=-\sqrt{-\frac{\alpha m^{2} \lambda^{2}-2 \beta}{\lambda^{2}}} t+m x$, and $C$ is an arbitrary constant.

Case IV \& Case V:

When $\lambda^{2}-4 \mu=0$, the executing value of $A_{0}$ is undefined. So the solution cannot be obtained. For this purpose Case IV is rejected.

Similarly when $\lambda^{2}-4 \mu=0, \mu=0, \lambda=0$ the executing value of $A_{0}, A_{1}$ are undefined. So the solution cannot be obtained. So Case $\mathrm{V}$ is also rejected.

\section{Results and discussions}

\subsection{Physical explanation}

In this part, we discus about the physical depiction of obtained and solitary wave solutions for the Klein-Fock-Gordon equation with respect to the $\exp (-\varphi(\xi))$-expansion method. Obviously the $3^{\text {rd }}$ order Klein-Fock-Gordon equation has solitary wave solutions that have exponentially decaying wings. There is difference type of traveling wave solutions that one of special interest in solitary wave theory. For some different physical parameters, solitary wave solutions are developed from the acquired exact solutions. Figure 1 represents Bright kink shape solution of $u_{1}$ for the parameters $\alpha=-2, \beta=-2, \delta=2, C=-1, m=2, \lambda=3, \mu=2$ within the interval $-10 \leq x, t \leq 10$. Figure 2 represents the Dark kink shape solution of $u_{2}$ for the parameters $\alpha=-2, \beta=-2, \delta=2, C=-1, m=2, \lambda=3, \mu=2$ within the interval $-10 \leq x, t \leq 10$. Figure 3 represents the Singular kink shape solution of $u_{3}$ for the parameters $\alpha=-2, \beta=-2, \delta=1, C=-1, m=2, \lambda=3, \mu=2$ within the interval $-10 \leq x, t \leq 10$. Figure 4 represents the Periodic shape solution of $u_{6}$ for the parameters $\alpha=-4, \beta=-3, \delta=1, C=-1, m=2, \lambda=3, \mu=6$ within the interval $-10 \leq x, t \leq 10$ and Figure 5 represents the Soliton shape solution of $u_{10}$ for $\alpha=2, \beta=-2, \delta=-1, C=-1, m=2, \lambda=2, \mu=0$ within the same interval $-10 \leq x, t \leq 10$.

\subsection{Graphical explanation}

This sub-section represents the graphical representation of 3rd order Klein-Fock-Gordon equation. By using mathematical software Maple 18, Contour, 3D and 2D plots of some achieved solutions have been shown in Fig.-1-Fig.-5 to envisage the essential instrument of the original equations.
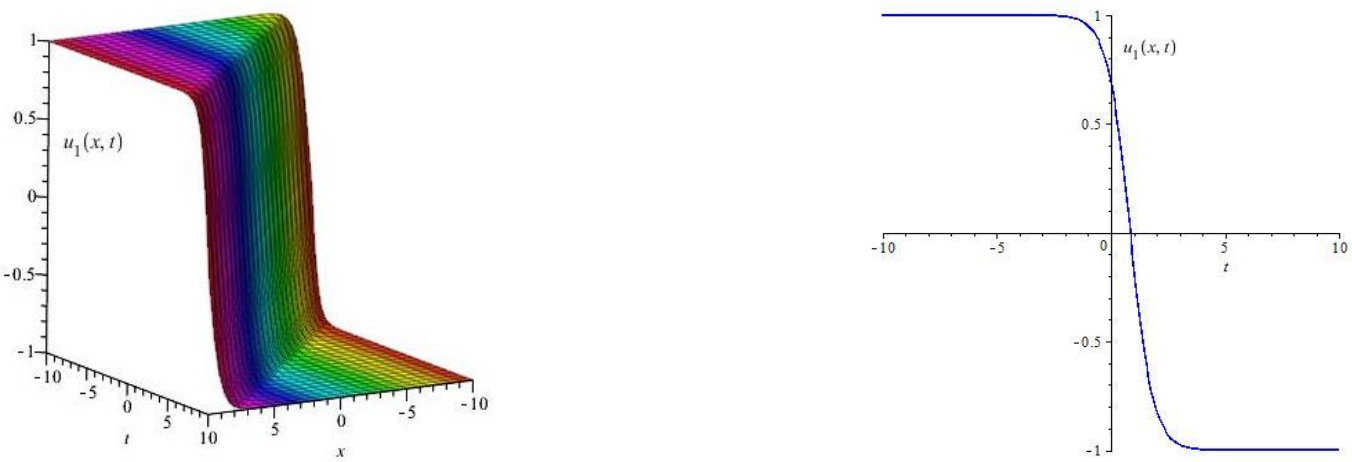

Fig. 1: Bright Kink Shape Solution of $u_{1}$ For $\alpha=-2, \beta=-2, \delta=2, C=-1, m=2, \lambda=3, \mu=2$ within $-10 \leq x \leq 10$ and $-10 \leq t \leq 10$. the Left Figure Shows the 3D Plot and the Right Figure Shows the 2D Plot for $x=1$. 

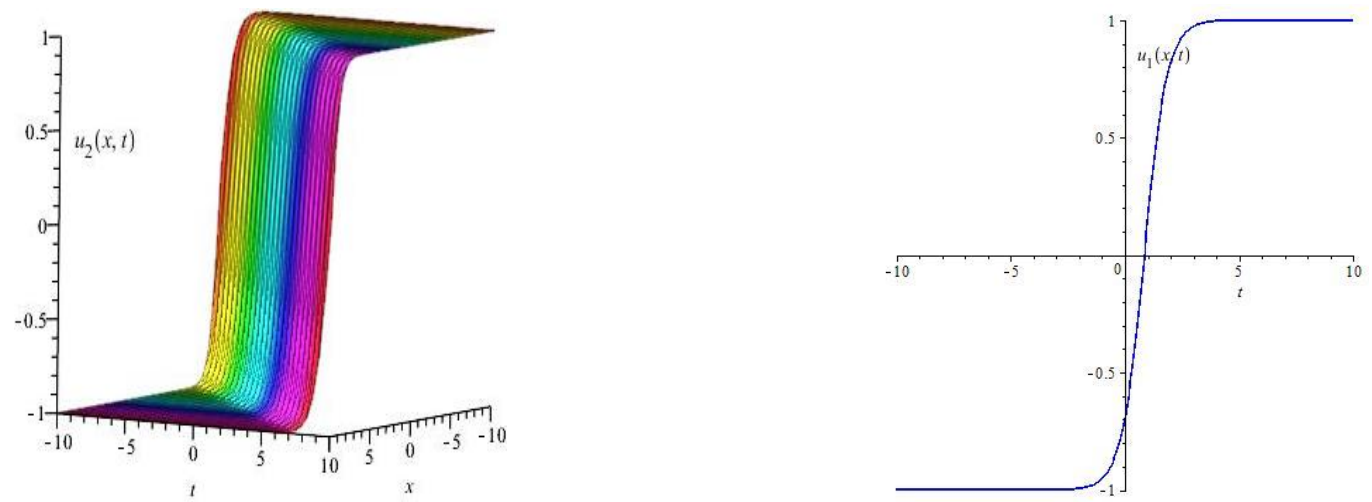

Fig. 2: Dark Kink Shape Solution of $u_{2}$ For $\alpha=-2, \beta=-2, \delta=2, C=-1, m=2, \lambda=3, \mu=2$. the Left Figure Shows the 3D Plot and the Right Figure Shows the 2D Plot for $x=1$.
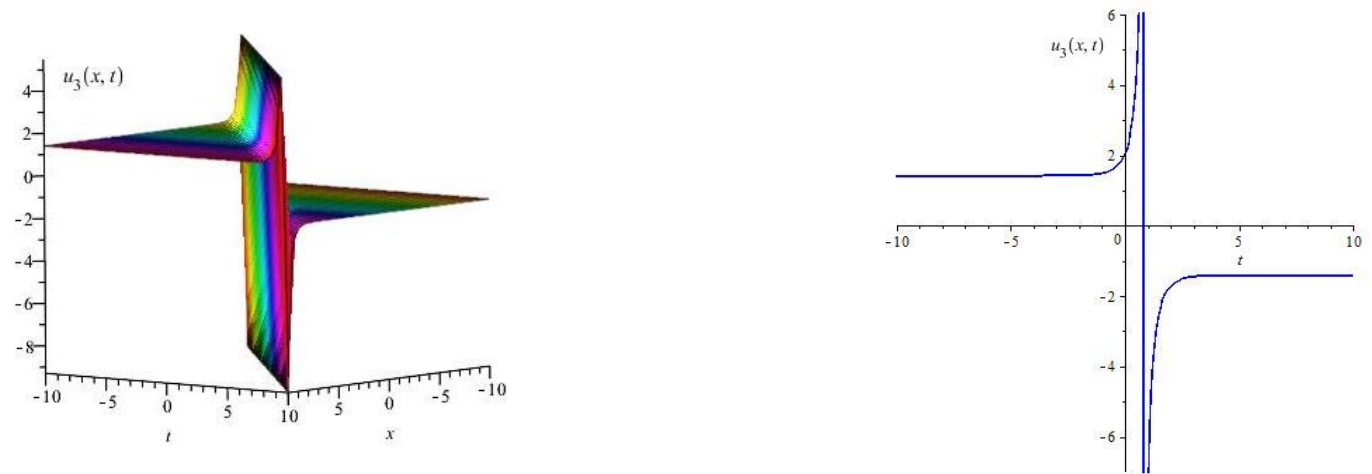

Fig. 3: Singular Kink Shape Solution of $u_{3}$ For $\alpha=-2, \beta=-2, \delta=1, C=-1, m=2, \lambda=3, \mu=2$. the Left Figure Shows the 3D Plot and the Right Figure Shows the 2D Plot for $x=1$.
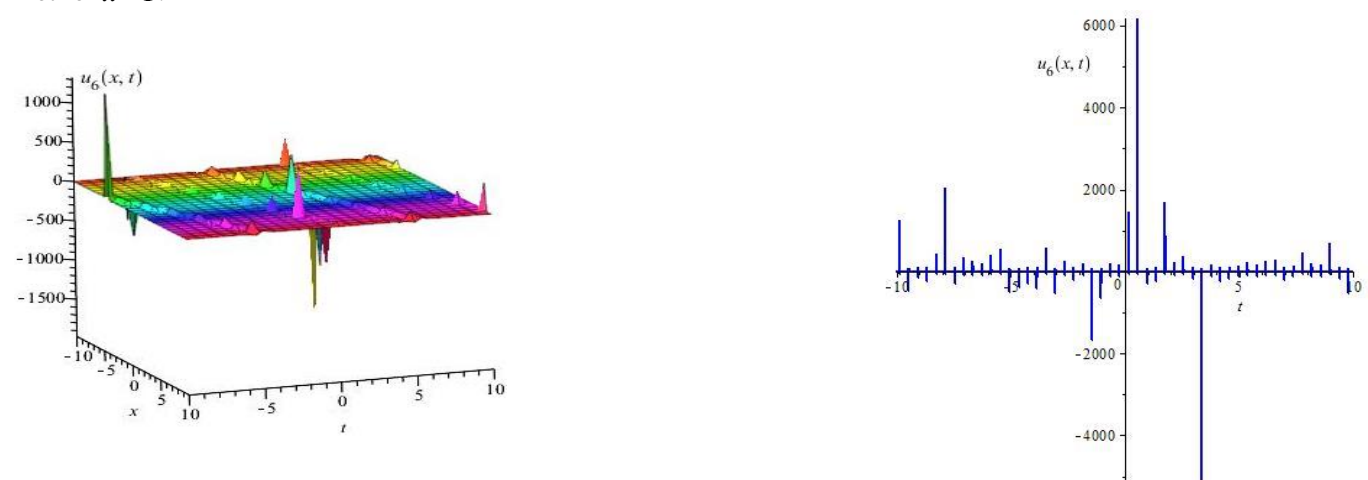

Fig. 4: Periodic Shape Solution of $u_{6}$ for $\alpha=-4, \beta=-3, \delta=1, C=-1, m=2, \lambda=3, \mu=6$. the Left Figure Shows the 3D Plot and the Right Figure Shows the 2D Plot for $x=1$.

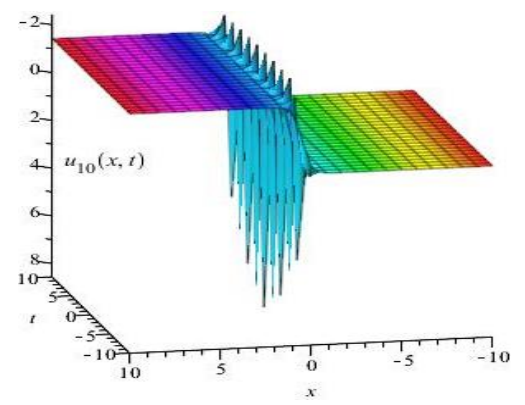

Fig. 5: Soliton Shape Solution of $u_{10}$ for $\alpha=2, \beta=-2, \delta=-1, C=-1, m=2, \lambda=2, \mu=0$.

\section{Conclusion}

In this study, the $\exp (-\varphi(\xi))$-expansion has been successfully applied to find new traveling wave solutions for nonlinear wave equation of $3^{\text {rd }}$-Order Klein-Fock-Gordon equation (KFGE). We get some new traveling wave solutions including hyperbolic function solutions, trigonometric function solutions and exponential solutions. Therefore, we successfully got the Bright kink shape solution, Dark kink shape solution, Singular kink shape solution, Periodic shape solution and other Soliton shape solution which has given the proper geometrical explanation. The results is clear to us that our proposed method is reliable, effective and reasonable good for nonlinear evolution equations. 
Likewise, the solutions of the proposed nonlinear evolution equation in this paper have numerous potential applications in engineering field.

\section{Acknowledgement}

The authors would like to thank the researcher of supported journal of nonlinear field.

\section{Funding}

No funding.

\section{Competing interests}

The authors declare that they have no competing interests.

\section{Author's contributions}

All authors contributed equally to this work. All authors read and approved the final manuscript.

\section{References}

[1] Nakamura, Akira. "Surface Impurity Localized Diode Vibration of the Toda Lattice: Perturbation Theory Based on Hirota's Bilinear Transformation Method." Progress of Theoretical Physics 61.2 (1979): 427-442. https://doi.org/10.1143/PTP.61.427.

[2] Hietarinta, Jarmo. "A search for bilinear equations passing Hirota's three-soliton condition. I. KdV-type bilinear equations." Journal of Mathematical Physics 28.8 (1987): 1732-1742. https://doi.org/10.1063/1.527815.

[3] Khan K, Akbar MA. Application of the $\exp (-\Phi(\xi))$-expansion method to find the exact solutions of modified Benjamin-Bona-Mahony equation. World Appl. Sci.J.2013;24(10):13731377.

[4] Zhao, Mm, Li,C.The exp(- $\Phi(\xi))$-expansion method applied to nonlinear evolution equations; 2008.

[5] Roshid HO, Rahman MA. The exp(- $\Phi(\eta))$-expansion method with application in the (1+1)-dimensional classical Boussinesq equations. Results Phys; 2014. https://doi.org/10.1016/j.rinp.2014.07.006.

[6] He, Yinghui, Shaolin Li, and Yao Long. "Exact solutions of the Klein-Gordon equation by modified Exp-function method." Int. Math. Forum. Vol. 7. No. 4. 2012

[7] Kumar, Dipankar, and Melike Kaplan. "New analytical solutions of (2+ 1)-dimensional conformable time fractional Zoomeron equation via two distinct techniques." Chinese journal of physics 56.5 (2018): 2173-2185. https://doi.org/10.1016/j.cjph.2018.09.013.

[8] Khater, Mostafa M. A., and Emad HM Zahran. "New Method to Evaluating Exact and Traveling Wave Solutions for Non-Linear Evolution Equations." J. Comput. Theor. Nanosci 12 (2015): 1-9.

[9] Abdou, M. A. "The extended tanh method and its applications for solving nonlinear physical models." Applied mathematics and computation 190.1 (2007): 988-996. https://doi.org/10.1016/j.amc.2007.01.070.

[10] Fan, Engui. "Extended tanh-function method and its applications to nonlinear equations." Physics Letters A 277.4-5 (2000): 212-218. https://doi.org/10.1016/S0375-9601(00)00725-8.

[11] Wang, Gang-Wei, and Tian-Zhou Xu. "Group analysis and new explicit solutions of simplified modified Kawahara equation with variable coefficients." Abstract and Applied Analysis. Vol. 2013. Hindawi, 2013. https://doi.org/10.1155/2013/139160.

[12] Wang, Gang-Wei, et al. "Singular solitons, shock waves, and other solutions to potential KdV equation." Nonlinear Dynamics 76.2 (2014): 10591068. https://doi.org/10.1007/s11071-013-1189-9.

[13] Wang, Gang-Wei, et al. "Solitons and Lie group analysis to an extended quantum Zakharov-Kuznetsov equation." Astrophysics and Space Science 349.1 (2014): 317-327. https://doi.org/10.1007/s10509-013-1659-z.

[14] Wang, Gang-wei, Xi-qiang Liu, and Ying-yuan Zhang. "New explicit solutions of the fifth-order KdV equation with variable coefficients." Bull. Malays. Math. Sci. Soc 37.3 (2014): 769-778.

[15] Jawad, Anwar Ja'afar Mohamad, Marko D. Petković, and Anjan Biswas. "Modified simple equation method for nonlinear evolution equations." Applied Mathematics and Computation 217.2 (2010): 869-877. https://doi.org/10.1016/j.amc.2010.06.030.

[16] Zayed, Elsayed ME. "A note on the modified simple equation method applied to Sharma-Tasso-Olver equation." Applied Mathematics and Computation 218.7 (2011): 3962-3964. https://doi.org/10.1016/j.amc.2011.09.025.

[17] Bai, Cheng-Lin, and Hong Zhao. "Complex hyperbolic-function method and its applications to nonlinear equations." Physics Letters A 355.1 (2006): 32-38. https://doi.org/10.1016/j.physleta.2006.01.094.

[18] Wang, Mingliang, Xiangzheng Li, and Jinliang Zhang. "Sub-ODE method and solitary wave solutions for higher order nonlinear Schrödinger equation." Physics Letters A 363.1-2 (2007): 96-101. https://doi.org/10.1016/j.physleta.2006.10.077.

[19] Wang, Deng-Shan, Yu-Jie Ren, and Hong-Qing Zhang. "Further extended sinh-cosh and sin-cos methods and new non traveling wave solutions of the (2+1)-dimensional dispersive long wave equations." Appl. Math. E-Notes 5 (2005): 157-163.

[20] Wang, Mingliang, Xiangzheng Li, and Jinliang Zhang. "The ( $\left.\mathrm{G}^{\prime} \mathrm{G}\right)$-expansion method and travelling wave solutions of nonlinear evolution equations in mathematical physics." Physics Letters A 372.4 (2008): 417-423. https://doi.org/10.1016/j.physleta.2007.07.051.

[21] Islam ME, Khan K, Akbar MA, Islam R. Traveling wave solutions of nonlinear evolution equation via enhanced (G'/G)-expansion method. GANIT J. Bangladesh Math. Soc. 2013; 33:83-92. https://doi.org/10.3329/ganit.v33i0.17662.

[22] Bekir, Ahmet. "Application of the (G' G)-expansion method for nonlinear evolution equations." Physics Letters A 372.19 (2008): $3400-3406$. https://doi.org/10.1016/j.physleta.2008.01.057.

[23] Islam MH, Khan K, Akbar MA, Salam MA. Exact traveling wave solutions of modified KdV-Zakharov-Kuznetsov equation and viscous burgers equation. Springer Plus.2014;3:105. https://doi.org/10.1186/2193-1801-3-105.

[24] Ali, Ahmad T. "New generalized Jacobi elliptic function rational expansion method." Journal of computational and applied mathematics 235.14 (2011): 4117-4127. https://doi.org/10.1016/j.cam.2011.03.002.

[25] Fan, Engui, and Hongqing Zhang. "A note on the homogeneous balance method." Physics Letters A 246.5 (1998): 403-406. https://doi.org/10.1016/S0375-9601(98)00547-7.

[26] Wang, Mingliang. "Exact solutions for a compound KdV-Burgers equation." Physics Letters A 213.5-6 (1996): $279-287$. https://doi.org/10.1016/0375-9601(96)00103-X.

[27] Sharma, Dinkar, Prince Singh, and Shubha Chauhan. "Homotopy perturbation transform Method with He's polynomial for solution of coupled nonlinear partial differential equations." Nonlinear Engineering 5.1 (2016): 17-23. https://doi.org/10.1515/nleng-2015-0029. 
[28] Bayat, Mahmoud, Iman Pakar, and Ganji Domairry. "Recent developments of some asymptotic methods and their applications for nonlinear vibration equations in engineering problems: A review." Latin American Journal of Solids and Structures 9.2 (2012): 1-93. https://doi.org/10.1590/S167978252012000200003.

[29] Zayed, E. M. E., and Khaled A. Gepreel. "A series of complexiton soliton solutions for nonlinear Jaulent-Miodek PDEs using the Riccati equations method." Proceedings of the Royal Society of Edinburgh Section A: Mathematics 141.5 (2011): 1001-1015. https://doi.org/10.1017/S0308210510000405.

[30] He, Ji-Huan. "Variational iteration method for autonomous ordinary differential systems." Applied Mathematics and Computation 114.2-3 (2000): 115-123. https://doi.org/10.1016/S0096-3003(99)00104-6.

[31] Akbulut, Arzu, and Filiz Taşcan. "Application of conservation theorem and modified extended tanh-function method to (1+ 1)-dimensional nonlinear coupled Klein-Gordon-Zakharov equation." Chaos, Solitons \& Fractals 104 (2017): 33-40. https://doi.org/10.1016/j.chaos.2017.07.025.

[32] Hafez, M. G., Md Nur Alam, and M. Ali Akbar. "Exact traveling wave solutions to the Klein-Gordon equation using the novel (G'/G)-expansion method." Results in Physics 4 (2014): 177-184. https://doi.org/10.1016/j.rinp.2014.09.001. 